\title{
RECENT MANAGEMENT IN PATIENTS OF CARCINOMA RECTUM WITH RESPECT TO QUALITY OF LIFE- A RETROSPECTIVE AND PROSPECTIVE STUDY
}

\author{
Sanjay Kumar Mahajan', Rajkumar Mathur², Parvinder Lubana33, Sachin Verma ${ }^{4}$
}

${ }_{1}^{1}$ Senior Resident, Department of Surgery, MGM and MC, Indore, Madhya Pradesh.

2 Professor and HOD, Department of Surgery, MGM and MC, Indore, Madhya Pradesh.

${ }^{3}$ Consultant, Apple Hospital, Indore, Madhya Pradesh.

${ }^{4}$ Assistant Professor, Department of Surgery, MGM and MC, Indore, Madhya Pradesh.

ABSTRACT

\section{BACKGROUND}

Colorectal cancer is a major health problem worldwide. CRC remains in the top three of all cancer deaths and after its diagnosis and treatment the quality of life in patient of colorectal carcinoma is major morbidity issue worldwide, but the neoadjuvant treatment has come to overcome this morbidity in population. The aim of this review is to discuss important issues surrounding rectal cancer including its surgical and medical management with respect to quality of life.

The aim of this study was to assess the quality of life in carcinoma rectum patients undergoing recent management with neoadjuvant chemotherapy in MY Hospital and Cancer Hospital, Indore from Oct. 2011 to Sep. 2015.

\section{MATERIALS AND METHODS}

40 patients were treated with only surgical management and 26 patients were treated with neoadjuvant treatment followed by surgery retrospectively and prospectively followed up. All patients were treated in curative attempt. Quality of life was assessed 6 months after the surgery using anonymous questionnaire.

\section{RESULTS}

Main effect on the factors for the variables Physical function, Role function, Emotional function, Cognitive function and Social function show significant improvement in Neoadjuvant group. Both groups showed a major difference in Role function and a consistent increase in Emotional well-being and Social functioning perspective across the time of the study.

\section{CONCLUSION}

Based on the results of earlier studies of QoL cases undergoing Neoadjuvant treatment versus Non-Neoadjuvant and our own findings, we deduced that cases undergoing Neoadjuvant treatment do have some restriction in their post-treatment QoL such as nausea, vomiting and financial difficulties. Otherwise, their quality of life is better than patient not undergoing Neoadjuvant treatment and equally important is the fact that cases undergoing Neoadjuvant treatment have more benefit in QoL as better surgical correction and post-operative disease free survival is the most important outcome of our study.

\section{KEYWORDS}

Quality of Life, Neoadjuvant Treatment, EORTC-QLQ-C30, Carcinoma Rectum.

HOW TO CITE THIS ARTICLE: Mahajan SK, Mathur R, Lubana P, et al. Recent management in patients of carcinoma rectum with respect to quality of life- a retrospective and prospective study. J. Evolution Med. Dent. Sci. 2018;7(14):1689-1694, DOI: $10.14260 /$ jemds/2018/382

\section{BACKGROUND}

Colorectal cancer (CRC) is the third most commonly diagnosed cancers in males and the second in females with over 1.2 million new cancer cases.(1) In the past two decades incidence rates for CRC have remained largely unchanged, instead mortality rates have fallen due to improvements in early detection and cancer treatment.(2,3) Survival at 5 years is $56 \%$ in Europe and $66 \%$ in the United States of America. (4) Moreover, Baade et al concluded that survival expectations increase the longer they survive, reaching $93.2 \%$ at 5 years after diagnosis.(5) This leads to a rising prevalence of patient living with CRC with an estimated worldwide prevalence of

'Financial or Other Competing Interest': None.

Submission 28-02-2018, Peer Review 18-03-2018,

Acceptance 21-03-2018, Published 02-04-2018.

Corresponding Author:

Dr. Sanjay Kumar Mahajan,

\#180, Siddhipuram Colony,

Near Vaishali Nagar, Indore-452009, Madhya Pradesh.

E-mail: sanjaymahajan2211@gmail.com

DOI: $10.14260 /$ jemds $/ 2018 / 382$ more than 3 million persons within 5 years of diagnosis in 2008.(6) The rise of patients living with the consequence of CRC and its treatment has increased greatly the interest of their impact on health-related quality of life (QoL).(7) The loss of health due to the cancer and/or the consequence of the treatment may result in psychophysical or functional impairment or disruption of social and family interactions, all of these affect(8) QoL. Several studies assesses prospectively the impact of CRC in the patient's QoL, both in shortterm $(9,10)$ and long-term periods.

Preoperative chemoradiation (CRT) has been the standard of care in the United States for all patients with clinical stage II and III rectal cancer because of the low rates of local recurrence achieved,(11) acceptable levels of acute and late toxicity and the potential for sphincter preservation compared to postoperative chemoradiation. In parts of Northern Europe, a blanket approach to short course preoperative radiotherapy (SCPRT) using 25 Gy over 5 days followed by immediate surgery(12) within 2 - 5 days with the predominant aim of reducing the risk of pelvic recurrence has gained widespread acceptance. Recent improvements in the quality of surgery, magnetic resonance imaging and 
pathological reporting of the operative specimen, mean the time has come to question both these approaches. The majority of the rectum lies below the peritoneal reflection and has no serosa allowing tumour growth to extend deeply into perirectal fat. Historically, high rates of local pelvic recurrence following radical surgery were described. However, surgical practice has evolved and the technique of meticulous mesorectal dissection where the surgeon removes all of the surrounding mesorectal fat using sharp dissection in a neat anatomical package is associated with much lower rates of local recurrence and improved survival. With expert total mesorectal excision (TME) consistently performed in specialist centres, metastatic disease is now the predominant problem reflecting the likely presence of distant micrometastases at diagnosis, rather than inadequate surgery. It is true that old meta-analyses have shown that preoperative adjuvant radiotherapy reduces local recurrence rates by almost $50 \%$ and overall mortality by $2 \%-10 \%$. However, the local recurrence rates were very high in the region of $15 \%-30 \%$, and importantly the trials included in these meta-analyses all use patient's data from long before the introduction of total mesorectal excision (TME) surgery.(13)

Survival rates have increased throughout the last decades because of earlier diagnosis, improved diagnostic tests, introduction of adjuvant therapy and advances in the treatment of metastatic disease. Approximately, $80 \%$ of patients now survive the first year after diagnosis and approximately $62 \%$ survive 5 years and more.

Besides disease-free and overall survival time, quality of life (QOL) has become an important outcome measure for cancer patients.(14) The term quality of life refers to a multidimensional concept, which includes at least the dimensions of physical, emotional and social functioning. In addition, assessment of QOL in patients with cancer may improve our understanding of how cancer and therapy influence the patients' lives and how to adapt treatment strategies.(15)

Colorectal cancer and its treatment can have an adverse effect on social functioning including work and productive life; relationships with friends, relatives and partners; and other social activities and interests. Patients with colorectal cancer, both stoma and non-stoma patients, are troubled by frequent or irregular bowel movements, diarrhoea, flatulence, fatigue and often have to follow dietary restrictions. Comparisons between cancer survivors and healthy controls provide evidence that cancer survivors face ongoing problems related to the cancer disease including reduction in energy, weight loss and psychological distress.(16) Less is known, however, about how colorectal cancer patients rate their overall QOL and emotional and social functioning and how they cope with the awareness of living with a chronic and potentially life-threatening disease. We, therefore, initiated a study to describe the QOL of patients with colorectal cancer after diagnosis when acute treatment effects are expected to have declined.

\section{Aims and Objectives}

1. To assess the management to improve Quality of Life in case of carcinoma rectum patients undergoing neoadjuvant treatment.
2. To assess Quality of Life after the surgical management of colorectal carcinoma.

\section{Inclusion Criteria}

1. Patients who are diagnosed as a case of carcinoma rectum, aged 18 years to 65 years of age.

2. Patients whose follow-up is possible.

3. Patients who give valid informed written consent.

\section{Exclusion Criteria}

1. Patients having malignancy at other sites along with carcinoma rectum.

2. Morbid or inoperable patient.

3. Patient who were not given valid informed written consent.

\section{MATERIALS AND METHODS}

For this study the material consisted of 66 patients, more than 18 yrs. of age up to 65 years of age, treated in Surgical Ward and Cancer Department of MY Hospital and MGM Medical College, Indore from January 2011 to October 2015.

A Retrospective and Prospective Study patients from a convenience sample, a group of 26 patients undergoing Neoadjuvant and a group of 40 patients undergoing Non Neoadjuvant and only surgical management were selected for the investigation. Inclusion criteria were as follows: Who are diagnosed as a case of carcinoma rectum Stage II and Stage III and aged more than 18 years of age. Patients whose follow-up are possible, Patients who gave valid informed written consent and both clinical and QoL data had to be available at each point of assessment. In this article we do not report on the clinical data, instead we focus on the QoL findings postoperatively after 6 months of surgery.

\section{Instruments}

Questionnaires were used to collect clinical and QoL data. Clinical information included tumour stage, surgical technique and tumour recurrence. QoL data were obtained using the European Organisation for Research and Treatment of Cancer (EORTC) QLQ C30 questionnaire (version 2.0, EORTC Study Group on quality of life). These are wellestablished tools for the assessment of QoL in patients with cancer. This have been proven reliable and valid. For almost two decades, the QLQ C30 instrument has been used to measure QoL in various samples of patients with cancer. These include five functional scales (Physical, Role, Cognitive, Emotional and Social), three symptom scales (Fatigue, Pain, Nausea and Vomiting), a global health status/ QoL scale and a number of single items assessing additional symptoms commonly reported by cancer patients (Dyspnoea, Loss of Appetite, Insomnia, Constipation and Diarrhoea).

\section{Procedure}

The study was prospective and retrospective. The assessment took place 6 months after surgery, Eligible patients were approached for the assessment when in the hospital awaiting the initiation of treatment or come for follow-up. They were informed of the goals of the study and the method of data collection and were invited to participate in the study, but also assured that their refusal would not jeopardise their 
treatment at the hospital. Informed consent was obtained from every patient who participated in the study. The patients were then administered the QoL questionnaire and their clinical data were recorded. They were given a QoL questionnaire that they could complete on the spot or fill out at home and return to the hospital; self-addressed stamped envelopes were provided. At assessment, relevant clinical data were obtained by the treating physician.

\section{General Principles of Scoring}

The QLQ-C30 is composed of both multi-item scales and single-item measures. These include five functional scales, three symptom scales, a global health status/ QoL scale and six single items.

Each of the multi-item scales included a different set of items- no item occurs in more than one scale.

All of the scales and single-item measures range in score from 0 to 100. A high scale score represents a higher response level.

Thus, a high score for a functional scale represents a high/healthy level of functioning, a high score for the global health status/QoL represents a high QoL, but a high score for a symptom scale/item represents a high level of symptomatology/problems.

\section{Data Collection and Analysis}

EORTC-QLQ C-30 (European Organisation for Research and Treatment of Cancer) was utilised to assess the QOL Mean and Median of demographic data was done by using chisquare and independent ' $\mathrm{t}$ ' test. Statistical analysis was performed using SPSS 11.0.

\section{RESULTS}

The actual incidence of colorectal carcinoma is more in male as compared to female, but in our study, it was more in female $35(57 \%)$ as compared to male $31(43 \%)$. In this study, more number of cases were found in older age group, that is $28(42 \%)$ cases between 31 - 50 years, 27 (41\%) cases were more than 51 years and $12(18 \%)$ cases were found in younger age between 18 to 30 years out of total 66 cases. For socioeconomic status, it was seen that $43(66 \%)$ patients were from below poverty line and 23 (34\%) patients were from above poverty line status. We divided cases in two groups, i.e. the cases getting Neoadjuvant and cases not getting Neoadjuvant treatment. $26(39.4 \%)$ cases in the study underwent Neoadjuvant and 40 cases $(60.6 \%)$ were not getting any Neoadjuvant treatment. The Neoadjuvant group comprised of 11 men (42.3\%) and 15 women (57.6\%), the Non-Neoadjuvant group comprised of 20 men (50\%) and 20 women $(50 \%)$. There were no significant differences in age
( $\mathrm{T}$ test $=2.29, \mathrm{p}>.10$ ) in both the groups. The mean age was 43.77 years in the Neoadjuvant and 51.13 years in the NonNeoadjuvant. Possible subscale scores on each of the function and symptom scales can range from 0 to 100. A high score on a functional subscale indicates good functioning and little restriction in QoL; conversely, a high score on the symptom scales symbolises severe distress. Analysis of the QoL questionnaire yielded mostly significant results for the groups of Neoadjuvant treatment on most of the functional scales.

Case shows better functional score in both stage II ( $p=$ $0.0002)$ and Stage III $(p=.002)$. As expected cases belonging to APL shows significantly better functional score in Neoadjuvant than Non-Neoadjuvant group $(p=0.018)$, while in BPL group patient getting Neoadjuvant therapy also shows better functional score than Non-Neoadjuvant group $(\mathrm{p}=0.001)$.

In comparison to surgery in both surgery group APR and LAR, Neoadjuvant patient gave better result. In age specific factor related quality of life, older patient of Neoadjuvant group shows more symptomatic relief and better outcome as compared to Non-Neoadjuvant group. While unexpectedly, younger cases showed significantly more symptoms like pain $(\mathrm{df}=1, \mathrm{p}=0.02)$ and appetite loss than Non-Neoadjuvant group.

\begin{tabular}{|c|c|c|c|c|c|c|c|}
\hline & \multicolumn{3}{|c|}{$\begin{array}{c}\text { Getting } \\
\text { Neoadjuvant }\end{array}$} & \multicolumn{4}{c|}{$\begin{array}{c}\text { Not Getting } \\
\text { Neoadjuvant }\end{array}$} \\
\hline Variables & Mean & SD & Median & Mean & SD & Median & P Value \\
\hline QL & 77.17 & 9.80 & 75 & 56.95 & 19.46 & 50 & 0.001 \\
\hline PF & 76.23 & 9.39 & 73.3 & 59.34 & 16.37 & 60 & 0.01 \\
\hline RF & 73.20 & 10.92 & 66.7 & 56.11 & 27.16 & 66.7 & 0.01 \\
\hline EF & 72.83 & 10.12 & 75 & 59.72 & 18.19 & 58.3 & 0.00 \\
\hline CF & 89.86 & 13.97 & 100 & 71.66 & 24.43 & 66.7 & 0.00 \\
\hline SF & 70.31 & 9.98 & 66.7 & 58.33 & 22.22 & 66.7 & 0.02 \\
\hline FA & 17.37 & 14.52 & 11.1 & 24.06 & 18.24 & 22.2 & 0.15 \\
\hline NV & 19.57 & 18.56 & 16.7 & 1.67 & 5.10 & 0 & 0.00 \\
\hline PA & 23.17 & 14.84 & 33.3 & 22.76 & 19.31 & 33.3 & 0.93 \\
\hline DY & 2.90 & 9.59 & 0 & 8.88 & 17.36 & 0 & 0.48 \\
\hline SL & 10.13 & 15.67 & 0 & 23.32 & 23.41 & 33.3 & 0.02 \\
\hline AP & 23.17 & 15.67 & 33.3 & 29.99 & 26.77 & 33.3 & 0.28 \\
\hline CO & 8.69 & 18.02 & 0 & 16.66 & 20.99 & 0 & 0.15 \\
\hline DI & 23.17 & 21.16 & 33.3 & 24.44 & 26.17 & 33.3 & 0.85 \\
\hline FI & 52.18 & 19.69 & 66.7 & 47.78 & 24.29 & 50 & 0.48 \\
\hline \multicolumn{3}{|c|}{ Table 1. Comparison of Score for Quality } \\
& \multicolumn{8}{|c|}{ of Life in both the Groups } \\
\hline
\end{tabular}




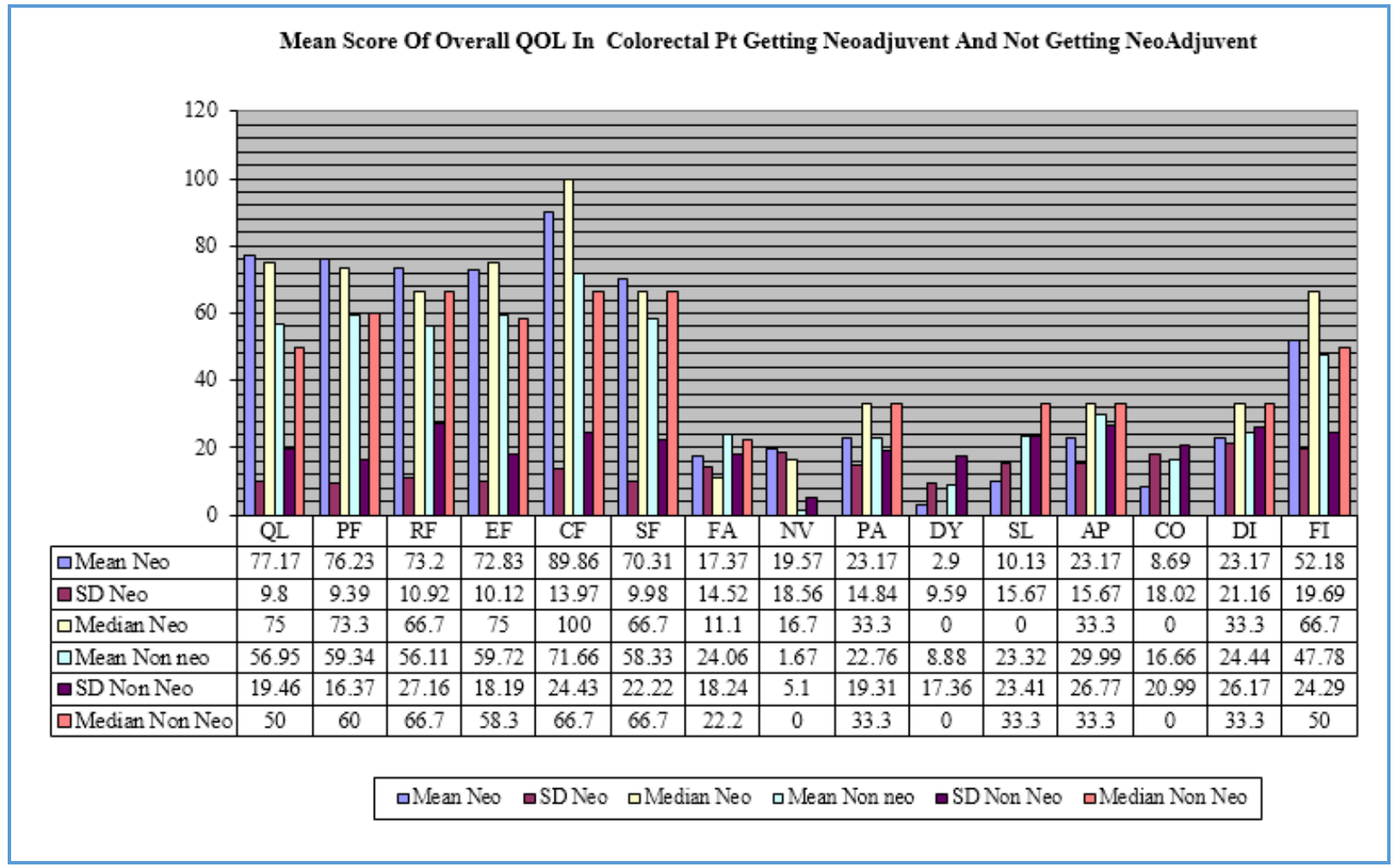

Figure 1

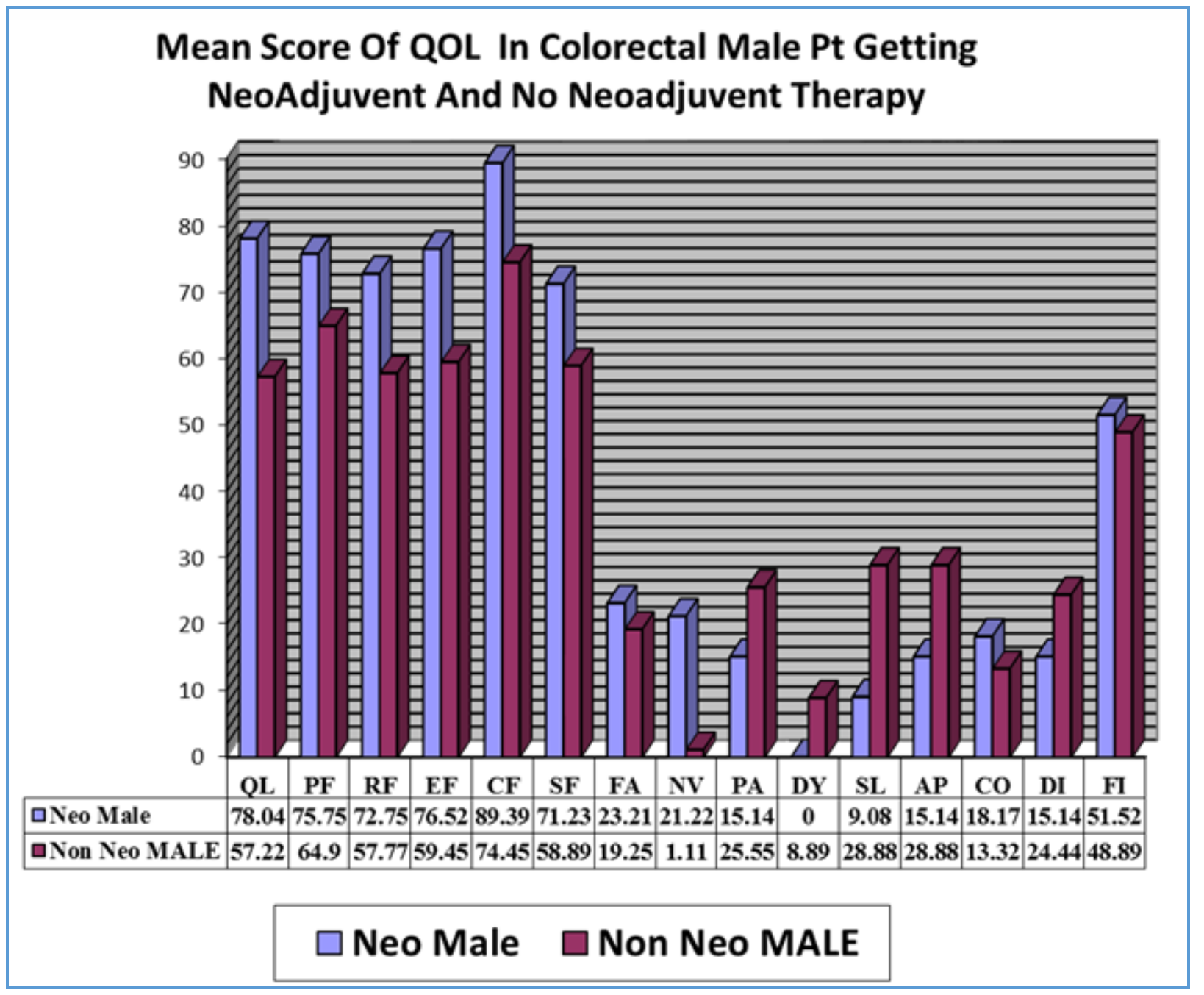

Figure 2 


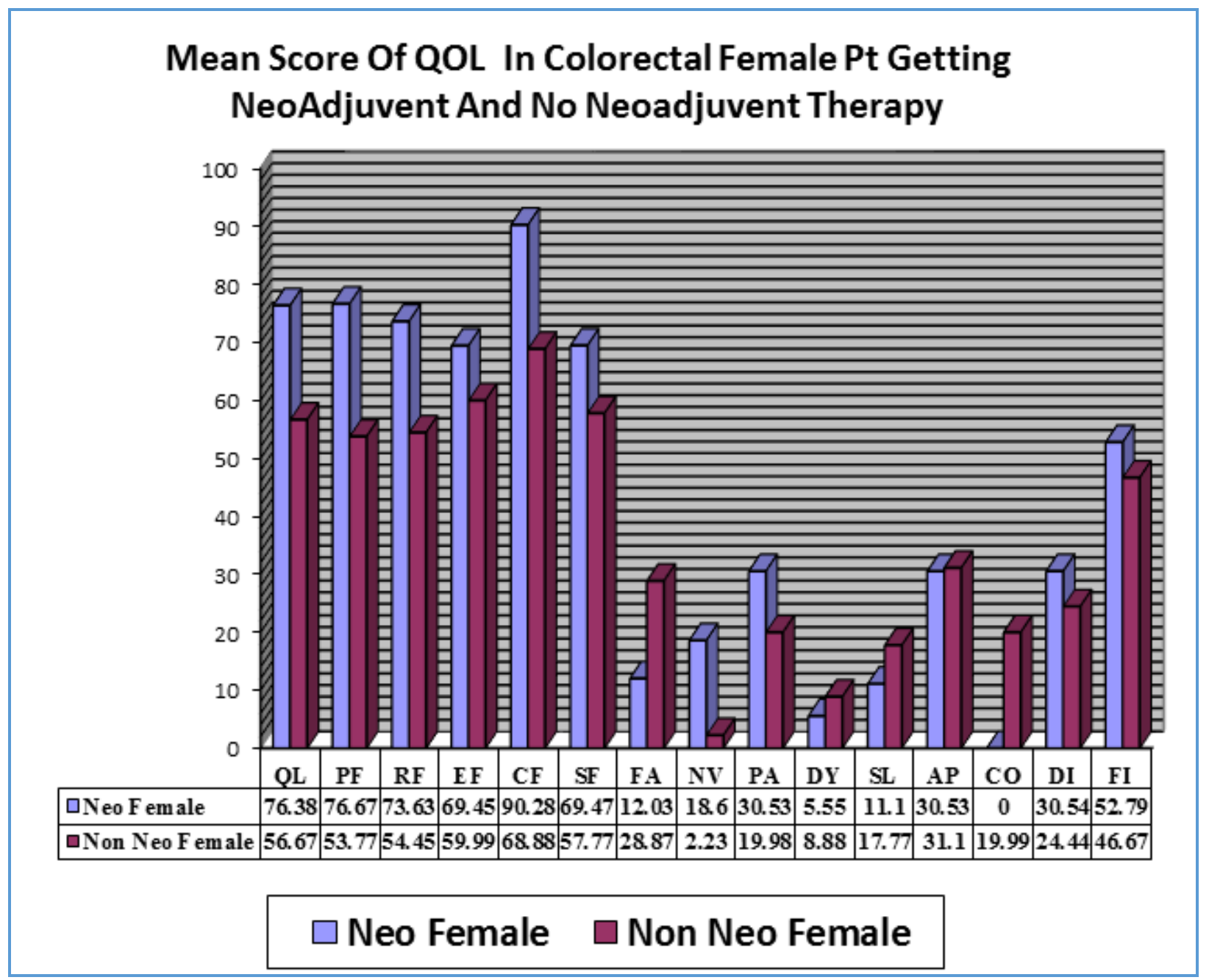

\section{DISCUSSION}

As expected, the Neoadjuvant cases showed a consistent tendency toward a better QoL than the Non-Neoadjuvant cases, especially those who are stage III and old age on most of the scales in the EORTC-QLQ-C30 questionnaire. The literature on the prominent concept of "fighting spirit" has revealed that cases with a fighting attitude may survive longer than cases who stoically accept their fate.(17) Neoadjuvant cases tended to exhibit superior physical, emotional, cognitive and social function, and reported less fatigue, sleeplessness, constipation and diarrhoea. Only for the subscale on Nausea, Vomiting and Financial Difficulty problems did Neoadjuvant cases score slightly less favourably.

The formulation of precisely selecting a patient group prevented the results from being distorted by clinical confounding factors (e.g. tumour spread, tumour localisation). A selection of cases with II and III tumour stages, who show no evidence of tumour recurrence throughout the study period might exemplify a subgroup of cases strongly determined to fight their cancer. Further, the strict research design limited the number of cases eligible for the study, which produced a small sample. Small group size complicates interpretation of the study findings. The small sample size is a critical point in our investigation calling for cautious conclusions at this stage. In line with numerous studies in the literature showing that cases with rectal cancer undergoing Neoadjuvant treatment have poor QOL,(18) our
Figure 3

group of Neoadjuvant treatment cases showed a consistent increase in QOL scores. Except cases undergoing Neoadjuvant treatment expressed the most pronounced problems with nausea, vomiting and financial difficulty. First, our findings confirmed with most of the evidence in the literature is not entirely novel. The Neoadjuvant Chemoradiotherapy study by Vivek Bansal, Ritu Bhutani, Dinesh Dova from Rohini Delhi shows better result in carcinoma rectum.(19)

Second, when attempting to embed our results in the body of psychological concepts and theories, Festinger's 30 theory of cognitive dissonance immediately comes to mind. This theory focuses on how beliefs and behaviour can change attitudes.(20) Although, Financial difficulty problems may well be a result of their difficulty in getting costly chemotherapy drugs and having more nausea and vomiting may be due to chemotherapy side effects.

\section{CONCLUSION}

Based on the results of earlier studies of QoL cases undergoing Neoadjuvant treatment versus Non-Neoadjuvant and our own findings, we deduce that cases undergoing Neoadjuvant treatment do have some restriction in their post-treatment QoL, such as Nausea vomiting and Financial difficulties. However, equally important is the fact that cases undergoing Neoadjuvant treatment have more benefit in QoL as compared to Non-Neoadjuvant treatment as better surgical correction and post-operative disease free survival is the most important outcome of our study. 
Our results should be used as an impetus to re-evaluate the most appropriate treatment option for each individual patient. In the past decades, tremendous progress in the development of modalities, techniques for the treatment of rectal cancer were made. Neo-adjuvant approaches are now used more frequently, which undoubtedly is a favourable development. However, we must not underestimate the cost of treatment on a patient's QoL. The stage downgrading and better surgical correction with disease free survival is the most important aspect to increase Quality of Life after Neoadjuvant treatment. However, we study only Chemotherapy as Neoadjuvant treatment in our study. The consequences of Radiotherapy still need to be evaluated and remains unanswered. The ultimate goal should be to determine, which patient benefits most from which type of treatment given his or her life circumstances.

\section{REFERENCES}

[1] Jemal A, Bray F, Center MM, et al. Global cancer statistics. CA: a Cancer Journal for Clinicians 2011;61(2):69-90.

[2] Biondi A, Grosso G, Mistretta A, et al. Laparoscopicassisted versus open surgery for colorectal cancer: short- and long-term outcomes comparison. Journal of Laparoendoscopic \& Advanced Surgical Techniques A 2013;23(1):1-7.

[3] Biondi A, Tropea A, Basile F. Clinical rescue evaluation in laparoscopic surgery for hepatic metastases by colorectal cancer. Surgical Laparoscopy, Endoscopy \& Percutaneous Techniques 2010;20(2):69-72.

[4] Verdecchia A, Francisci S, Brenner H, et al. Recent cancer survival in Europe: a 2000-02 period analysis of EUROCARE-4 data. The Lancet Oncology 2007;8(9):784-96.

[5] Baade PD, Youlden DR, Chambers SK. When do I know I am cured? Using conditional estimates to provide better information about cancer survival prospects. Medical Journal of Australia 2011;194(2):73

[6] GLOBOCAN. Cancer incidence, mortality and prevalence worldwide. http://www-dep.iarc.fr IARC Cancer Base No. 5. 2002: version 2.0.

[7] Donaldson GW, Moinpour CM. Individual differences in quality-of-life treatment response. Medical Care 2002;40(6 Suppl):III39-53.

[8] Frazzetto $P$, Vacante $M$, Malaguarner $M$, et al. Depression in older breast cancer survivors. BMC Surgery 2012;12(Suppl 1):S14.
[9] Smith-Gagen J, Cress RD, Drake CM, et al. Quality-oflife and surgical treatments for rectal cancer--a longitudinal analysis using the California Cancer Registry. Psychooncology 2010;19(8):870-8.

[10] Wilson TR, Alexander DJ, Kind P. Measurement of health-related quality of life in the early follow-up of colon and rectal cancer. Dis Colon Rectum 2006;49(11):1692-702.

[11] Sauer R, Becker H, Hohenberger W, et al. Preoperative versus postoperative chemoradiotherapy for rectal cancer. $\mathrm{N}$ Engl J Med 2004;351(17):1731-40.

[12] Capirci C, Valentini V, Cionini L, et al. Prognostic value of pathologic complete response after neoadjuvant therapy in locally advanced rectal cancer: long-term analysis of 566 ypCR patients. International Journal of Radiation Oncology Biology Physics 2008;72(1):99-107.

[13] Otto F, Lutz MP. Early gastrointestinal cancers II: rectal cancer. Springer 2014.

[14] Whynes DK, Neilson AR, Robinson MHE, et al. Colorectal cancer screening and quality of life. Qual Life Res 1994;3(3):191-8.

[15] Arndt V, Merx H, Stürmer T, et al. Quality of life among breast cancer survivors one year after diagnosis in comparison with women from the general population. Biometrical Journal 2004;46(1):102. DOI: 10.1002/bimj.200490005.

[16] Marventano S, Forjaz MJ, Grosso G, et al. Health related quality of life in colorectal cancer patients: state of the art. BMC Surgery 2013;13(Suppl 2):S15.

[17] Barrera S, Demark-Wahnefried W. Nutrition during and after cancer therapy. Oncology (Williston Park) 2009;23(Suppl 2):15-21.

[18] Schulze T, Wust P, Gellermann J, et al. Influence of neoadjuvant radiochemotherapy combined with hyperthermia on the quality of life in rectum cancer patients. Int J Hyperthermia 2006;22(4):301-18.

[19] Bansal V, Bhutani R, Doval D, et al. Neo adjuvant chemo-radiotherapy and rectal cancer: Can India follow the West? J Can Res Ther 2012;8(2):209-14.

[20] Fiske ST, Taylor SE. Cognitive approaches to attitudes. In: Fiske ST, Taylor SE. eds. Social Cognition. New York: McGraw-Hill 1991: p. 462509. 